\title{
AVALIAÇÃO DA EFICÁCIA DE UM PROGRAMA LUDOMOTOR DE EXERCICIOS FISICOS \\ NA MELHORA DA CAPACIDADE FUNCIONAL DE IDOSOS
}

\author{
Alisson Padilha de Lima ${ }^{1}$ \\ Fabrício Bruno Cardoso²
}

resumo

Este estudo teve como objetivo avaliar a eficácia de um programa ludomotor voltado para idosos que expressam corporalmente a dificuldade em executar com destreza, em termos de rapidez e coordenação, algumas atividades da vida diária que exijam de sua capacidade funcional. $\bigcirc$ universo deste estudo foi composto por 100 idosos de ambos os sexos com idade compreendida entre 72 e 75 anos, cadastrados no programa "Idoso Feliz" na cidade do Rio de Janeiro. Para a consecução do objetivo da pesquisa os participantes foram submetidos ao Protocolo de Habilidades Manuais (PHM), logo em seguida os participantes foram submetidos ao Teste de Coordenação (COO). Pode-se obter resultados significantes através das comparações do protocolo de habilidades manuais onde se apresentou um desempenho médio igual a 11,71 segundos no pré-teste e um desempenho de 10,60 segundos no pós-teste, já no teste de coordenação se obteve um escore médio de 11,29 segundos no pré-teste e um escore de 9,84 segundos no pós-teste, mostrando a eficiência da intervenção através do programa de exercício físico proposto a esses indivíduos. Dessa forma, pode-se afirmar a eficácia de um programa ludomotor de exercícios físicos na melhora da integração neuromotora do idoso em executar com destreza tarefas que exijam habilidades manuais e capacidades coordenativas, onde os mesmos obtiveram um feedback positivo da melhora dos seus índices de percepção de execução com êxito das tarefas impostas

1 Professor Bacharel e Licenciado em Educação Física pelo CEULJI/ULBRA, Especialista em Educação Especial pela UCDB, Personal Trainer para Populações Especiais com ênfase maior em idosos, Pesquisador do Laboratório de Temas Filosóficos em Conhecimento Aplicado (LABFILC) da Universidade Estadual do Rio de Janeiro UERJ. Departamento de Ciências da Atividade Física. E-mail: professor.alissonpadilha@gmail.com

2 Professor Mestre em Ciência da Motricidade Humana, Pesquisador do Laboratório de Temas Filosóficos em Conhecimento Aplicado (LABFILC) da Universidade Estadual do Rio de Janeiro UERJ. Departamento de Ciências da Atividade Física. E-mail: fabriciobrunocardoso@gmail.com 


\section{Introdução}

O processo de envelhecimento é caracterizado por diversas alterações. Dentre os fatores que interferem no processo de senescência estão os aspectos ambientais e a saúde física e mental, o que leva a efeitos deletérios na qualidade de vida, levando a uma desestruturação orgânica mais rápida e afetando, assim, a aptidão funcional do idoso (LIMA; DELGADO, 2010).

Algumas características decorrentes do envelhecimento humano alteram e causam mudanças no estilo de vida de indivíduos idosos, principalmente nas suas qualidades físicas, o que afeta a parte funcional desses indivíduos. Através dos efeitos benéficos de um programa de exercício físico, pode-se melhorar e prevenir a diminuição da aptidão funcional do idoso e suas respectivas qualidades físicas utilizadas em suas atividades da vida diária, como as capacidades coordenativas e as habilidades manuais (BRUIN et al., 2007).

Um dos maiores fatores que influenciam na qualidade de vida durante o processo de senescência é a interrupção da prática de atividade física, onde os indivíduos idosos são afetados pelos processos deletérios do envelhecimento, acarretando grandes perdas na capacidade funcional e interferindo profundamente nas suas atividades da vida diária, o que proporciona sérios problemas patológicos na vida desses indivíduos (PHILLIPS et al., 2010).

A importância de intervenções através de programas de exercícios físicos pode proporcionar, durante o processo de envelhecimento, uma série de efeitos benéficos na independência funcional, o que vem acarretar uma melhora na qualidade de vida, nas atividades da vida diária e obtendo uma melhora nas capacidades coordenativas e manuais desses indivíduos, o que se torna, para o idoso, um estilo de vida imprescindível para se obter dímidios agravantes a sua saúde (NICOLAI et al., 2010).

Dessa forma, a aplicação prática de um programa neuromotor de exercício físico bem elaborado beneficia os idosos nas suas capacidades coordenativas, permitindo a esses indivíduos assumirem a consciência da execução 
dos movimentos, levando ao encontro de uma integração progressiva de aquisições e favorecendo uma ação dos diversos grupos musculares, com vista à realização de movimentos com o máximo de eficiência (LIMA et al., 2011).

Diversos estudos comprovam a eficácia de um programa de exercício físico na população idosa e a sua prática influencia muito no estilo de vida desses indivíduos pelo fato de favorecer inúmeros benefícios na capacidade funcional, como: melhoras na coordenação em realizar movimentos da atividade da vida diária, execução de recursos de deambulação sozinhos, além da obtenção uma saúde vigorosa para se realizar tarefas como a prática de exercícios por mais tempo (GALLAGHER et al., 2010).

A partir do comentado anteriormente, este estudo teve como objetivo avaliar a eficácia de um programa ludomotor voltado para idosos que expressam corporalmente a dificuldade em executar com destreza, em termos de rapidez e coordenação, algumas atividades da vida diária que exijam de sua capacidade funcional.

\section{Métodos}

\subsection{Participantes do Estudo}

O universo deste estudo foi composto por 100 idosos de ambos os sexos com idade compreendida entre 72 e 75 anos, cadastrados no Programa "Idoso Feliz" na cidade do Rio de Janeiro/RJ. Para critérios de inclusão no estudo, eles deveriam ter cognição preservada, que foi atestada por obter pontuação acima de 24 pontos no Mini-Exame do Estado Mental, além disso, como critérios de exclusão, não poderiam apresentar doenças neurológicas crônicas, músculo-esqueléticas, vasculares e doenças com alteração da sensibilidade, como a hanseníase. Doenças, essas, detectada via exames clínicos. 
O projeto desta pesquisa foi submetido ao Comitê de Ética em Pesquisa envolvendo Seres Humanos do Centro Universitário Luterano de Ji-Paraná e aprovado sob protocolo n⿳0 $189 / 09$.

A coleta de dados se deu conforme a resolução 196/96. Assim, inicialmente foi solicitada a autorização dos participantes para que o estudo fosse desenvolvido, através de um Termo de Consentimento Livre e Esclarecido, ao qual se anexou uma carta de esclarecimentos explicando-se a natureza do estudo.

\subsection{Procedimentos de Avaliação}

Inicialmente os participantes deste estudo foram submetidos ao Protocolo de Habilidades Manuais (PHM) proposto por Andreotti e Okuma (1999). Este instrumento é formado de um painel retangular $(60 \mathrm{~cm}$ de comprimento e $15 \mathrm{~cm}$ de altura) com $5 \mathrm{~cm}$ de bordas inferior e laterais, contendo uma fechadura, uma tomada, um soquete para encaixar a lâmpada e um disco de telefone, dispostos horizontalmente com uma distância de $10 \mathrm{~cm}$ entre cada objeto e possuindo uma base perpendicular medindo $10 \mathrm{~cm}$ de largura; cronômetro, chave, plug e lâmpada. A testada, então, deveria colocar a chave na fechadura, colocar o plug na tomada, desencaixar a lâmpada do soquete e discar o número 9 no disco do telefone em menor tempo possível. Para tal, podia fazer três tentativas, das quais a tentativa de menor tempo era a registrada.

Logo em seguida os participantes foram submetidos ao Teste de Coordenação (COO) proposto por Osness et al. (1990), que consiste em um pedaço de fita adesiva com 76,2 cm de comprimento, fixada sobre uma mesa. Sobre a fita são feitas seis marcas com 12,7 cm eqüidistantes entre si, com a primeira e última marcas a $6,35 \mathrm{~cm}$ de distância das extremidades da fita. Sobre cada uma das seis marcas é afixado, perpendicularmente à fita, um pedaço de fita adesiva com 7,6 cm de comprimento, formando assim seis pequenos quadrados sobre a fita maior. $O$ sujeito senta-se de frente para a mesa e usa sua mão dominante para realizar o teste. Se a mão dominante for a direita, uma lata de refrigerante é colocada no quadrado 1, a lata dois no quadrado 3 e a lata três no quadrado 5. A mão direita será colocada na lata 1, com o polegar para cima, estando o cotovelo flexionado num ângulo de 100 a 120 graus. 
Quando o avaliador sinalizar, um cronômetro será acionado e o sujeito, virando a lata, inverte sua base de apoio, de forma que a lata um seja colocada no quadrado 2; a lata dois no quadrado 4 e a lata três no quadrado 6. Sem perda de tempo, o avaliado, tendo o polegar apontado para baixo, apanha a lata 1 e inverte novamente sua base, recolocando-a no quadrado 1 e, da mesma forma, procede colocando a lata dois no quadrado 3 e a lata três no quadrado 5, completando assim um circuito. Uma tentativa equivale à realização do circuito duas vezes, sem interrupções. O cronômetro é parado quando a lata 3 for colocada no quadrado 5, ao final do segundo circuito. Caso o sujeito seja canhoto, o mesmo procedimento será adotado, exceto que as latas serão colocadas a partir da esquerda: lata um no quadrado 6, lata dois no quadrado 4, lata três no quadrado 2 e assim por diante. A cada sujeito foram concedidas duas tentativas de prática, seguidas por outras duas válidas para avaliação, sendo - nestas últimas duas - anotados até os décimos de segundo, considerando como resultado final o menor dos tempos obtidos.

\subsection{Procedimentos de Intervenção}

A operacionalização do programa ludomotor ocorreu em 40 sessões com duração de 15 minutos, com periodicidade de três vezes na semana, através de exercícios funcionais com objetivo de auxiliar os idosos para que prolonguem por maior tempo possível sua independência funcional, considerando suas potencialidades e limitações na realização de suas atividades da vida diária (AVD) que necessitam de uma habilidade motriz manual. Para um melhor entendimento, descrevemos algumas das atividades desenvolvidas:

- Construir Petecas: Os idosos receberam, da equipe de pesquisadores, duas folhas de papel jornal medindo $20 \mathrm{~cm} \times 20 \mathrm{~cm}$, e cada participante confeccionou uma bola amassando uma folha do papel jornal, com objetivo de formar a base da peteca. Depois de amassar bem a base da peteca, o participante era orientado a pegar sua outra folha de papel jornal para cobrir a base da peteca com ela, porém devendo puxar todas as suas pontas para cima e juntando-as.

- Montar Quebra-Cabeça: A equipe de pesquisadores distribuiu para cada participante figuras a serem coloridas em um papel A4. Após colorirem, deveriam recortá-las em vários pedaços, posteriormente trocar as figuras entre os demais participantes e, por fim, montar a figura que lhe foi entregue. 
- Torre Flutuante: O participante deveria montar uma torre com 5 latas de refrigerante vazias sobre um prato plástico flutuando em uma tina de água, no tempo limite de 40 segundos.

- Passar Agulha: Utilizando apenas uma das mãos, o participante precisaria transpassar os buracos de 10 agulhas no limite de 120 segundos.

- Enroscar e desenroscar tampas de garrafas: o participante deveria enroscar e desenroscar tampas de 10 garrafas de $600 \mathrm{ml}$ no limite de 60 segundos.

\subsection{Tratamento Estatístico dos Dados}

A análise dos dados deste estudo foi realizada com base na comparação de resultados estatísticos utilizando-se o programa de estatística BIOESTAT 5.0 Os resultados obtidos nas avaliações do Protocolo de Habilidades Manuais (PHM) e do Teste de Coordenação (COO) dos idosos participantes deste estudo foram inicialmente calculados através da estatística descritiva, a média e o desvio padrão.

Para a comparação num sentindo pré-teste $X$ pós-teste, os resultados obtidos foram testados para a verificação de sua normalidade pelo teste de Lilliefors, que classificou como não-paramétricos os resultados obtidos em relação ao PHM e como paramétricos os resultados obtidos em relação ao COO.

De acordo com os resultados obtidos no teste de normalidade, optou-se, para a comparação entre as avaliações acerca do PHM, a utilização do teste de WILCOXON; em relação às avaliações do COO, utilizou-se o teste T-STUDENT. Cabe ressaltar que em ambas as comparações fora estabelecido o nível de significância de 5,00\%, o que configura uma certeza mínima na tomada de decisão de $95,00 \%$.

\section{Resultados}

A partir da tabela 1 é possível perceber os escores apresentados pelos idosos nas avaliações do Protocolo de Habilidades Manuais (PHM), onde se constatou um desempenho médio igual a 11,71 segundos no pré-teste e um desempenho médio de 10,60 segundos no pós-teste; cabe ressaltar que quanto menor for o tempo para a realização das tarefas, melhor é o desempenho do avaliado. 
Tabela 1 - Resultados dos idosos no PHM, nas duas avaliações.

\begin{tabular}{ccc}
\hline Categorias & $\mathbf{1}^{\mathbf{a}} \mathbf{A V}$ & $\mathbf{2}^{\mathbf{a}} \mathbf{A V}$ \\
\hline Mínimo & $9.43 \mathrm{~s}$ & $9.01 \mathrm{~s}$ \\
\hline Máximo & $13.75 \mathrm{~s}$ & $12.68 \mathrm{~s}$ \\
\hline Média Aritmética & $11.71 \mathrm{~s}$ & $10.60 \mathrm{~s}$ \\
\hline Desvio Padrão & 0.84 & 0.86 \\
\hline Coeficiente de Variação & $7.17 \%$ & $8.18 \%$ \\
\hline
\end{tabular}

AV: avaliação; s: segundos

Ao observar a tabela 1, nota-se uma diferença de 1,11 segundos entre as médias dos resultados dos idosos nas duas avaliações do $\mathrm{COO}$, diferença esta que, através do teste de WILCOXON, mostrou-se significante, pois para um $Z=8,68$ obteve-se um $p=0,01$, o que mostra que a evolução no desempenho dos idosos no referido teste não se deu ao acaso.

- Quando avaliados no COO inicialmente (1 ${ }^{\underline{a}}$ Avaliação), o desempenho dos idosos variou entre 9,77 segundos e 12,76 segundos, com um desempenho médio igual a 11,29 segundos (Tabela 2). Desempenho esse que, na segunda avaliação, foi igual a 9,84 segundos - pois a variação de resultados ficou entre 8,02 segundos e 11,88 segundos -, o que mostra uma tendência à evolução dos indivíduos avaliados após a intervenção proposta neste estudo, comprovada através do teste T-STUDENT, que para um $\mathrm{T}=29,38$ revelou um $p=0,02$.

Tabela 2 - Resultados dos idosos no COO nas duas avaliações.

\begin{tabular}{ccc}
\hline Categorias & $\mathbf{1}^{\mathbf{a}} \mathbf{A V}$ & $\mathbf{2}^{\mathbf{a}} \mathbf{A V}$ \\
\hline Mínimo & $9.77 \mathrm{~s}$ & $8.02 \mathrm{~s}$ \\
\hline Máximo & $12.76 \mathrm{~s}$ & $11.88 \mathrm{~s}$ \\
\hline Média Aritmética & $11.29 \mathrm{~s}$ & $9.84 \mathrm{~s}$ \\
\hline Desvio Padrão & 0.62 & 0.69 \\
\hline Coeficiente de Variação & $5.57 \%$ & $7.09 \%$
\end{tabular}

AV: avaliação; s: segundos 
Através das comparações do pré e pós-teste dos protocolos de habilidades manuais e de coordenação, foi constatado uma melhora nos escores dos idosos, o que mostrou a eficiência do programa de exercício físico proposto na intervenção desses indivíduos, levando-os a obter uma melhora nas destrezas de execução de movimento e, consequentemente, um maior índice de independência funcional para executar tarefas como as atividades da vida diária.

O que vem a corroborar com os achados de Mathews et al. (2010): além da coordenação motora, as habilidades manuais exigidas durante o dia-a-dia na vida do idoso podem ser mantidas com a prática regular de atividade física, onde a melhora da capacidade funcional desse indivíduo maximizará sua independência nas AVD. O mesmo foi constatado no presente estudo através dos efeitos significativos das intervenções do programa ludomotor, mostrando assim sua eficácia.

Conforme as intervenções ludomotoras apresentadas na presente pesquisa, a melhora das capacidades coordenativas e das habilidades manuais proporcionou aos idosos uma melhor qualidade de vida na realização das suas AVD, fato esse que comprova o valor do exercício físico na vida desses indivíduos. Isso fora identificado no estudo de Nicolai et al. (2010), através de intervenções analisadas dia-a-dia, como variabilidade dos efeitos do exercício físico na capacidade funcional do idoso, levando-os a se tornarem cada vez mais independentes no seu estilo de vida adotado. Assim, a interrupção desse ciclo de prevenção, através desses programas de exercício físico, vem a acarretar perdas na independência do idoso e prejuízos nas respectivas capacidades coordenativas durante sua vida (PHILLIPS et al., 2010).

Em análise da coordenação motora dos idosos sob prática da intervenção generalizada se obteve melhoras nos índices, o que, segundo Dias e Duarte (2005) - corroborando com os achados do presente estudo -, comprova que quando a coordenação motora é exercitada, a mesma tende a minimizar o crescente declínio dessa habilidade, decorrente das inúmeras alterações do sistema orgânico no decorrer do processo de envelhecimento.

Assim, programas de intervenções através do exercício físico, como o do presente estudo, vêm colaborando para que a população idosa possa obter uma melhora na qualidade de vida, nas capacidades coordenativas e nas habilidades manuais em realizar suas tarefas com destreza, prevenindo, assim, diversos problemas patológicos que venham afetar a capacidade funcional do idoso, através de seus inúmeros benefícios à saúde (SIVAN; SAWYER; 
BROWN, 2010). Benefícios esses que, conforme os achados de Lima et al. (2011), interferem na interação neuromotora do idoso através da integração das capacidades físicas em realizar suas atividades, e o leva a um melhor feedback na percepção da execução da tarefa com êxito, fato esse comprovado devido aos fatores de amostragem do estudo serem amplos e os testes aplicados com maximização de eficácia.

Outra pesquisa realizada com idosos vem ao encontro do presente estudo, onde se identificou a eficácia de intervenções com exercícios físicos na melhora das capacidades coordenativas: os níveis de coordenação de idosos foram melhorados com o engajamento na prática de atividade física no decorrer do processo de envelhecimento, a fim de se obter uma retroalimentação de hábitos adequados na maximização da capacidade funcional e, consequentemente, no aumento da qualidade de vida dessa população idosa (COHEN-MANSFIELD; SHMOTKIN; GOLDBERG, 2010).

Através da ginástica recreativa, Monte et al. (2006), após realização das intervenções propostas, observaram melhoras nos índices das capacidades coordenativas motoras dos idosos. Esse método vem, portanto, ao encontro do presente estudo, que obteve melhora nos índices após intervenção de um programa de exercício, mostrando a eficácia do mesmo na minimização dos efeitos deletérios do envelhecimento e proporcionando uma melhora na capacidade funcional desses indivíduos.

Já em outro programa de intervenção, Domingues e Santos (2006), através da ginástica rítmica, obtiveram o mesmo êxito do presente estudo, melhorando os níveis de destreza das habilidades manuais e proporcionando, através de um programa de exercício físico, que esses indivíduos executassem com destreza as suas atividades da vida diária que exijam uma integração maior do sistema motor. Contribuíram, assim, para uma relação mais fidedigna com os escores achados no presente estudo.

Dessa forma, torna-se plausível o efeito benéfico de uma intervenção adequada com um programa de exercício físico na melhora das habilidades manuais e nas capacidades coordenativas de idosos no decorrer do processo de senescência. Lee e Laffrey (2006) afirmam que, através da prática regular de exercício físico, podem ser minimizados agravantes à saúde do idoso e é possível aumentar sua expectativa de vida, proporcionando-lhe uma independência funcional ampla para se executar suas AVD. Já Cohen-Mansfield et al.(2006) afirmam que encontraram uma autoeficácia no exercício físico muito abrangente, que comprova a importância do engajamento desses indivíduos na prática regular de atividades físicas como diminuição desses índices complexos de perda funcional. 
Através da presente pesquisa, pode-se afirmar a eficácia de um programa ludomotor de exercícios físicos na melhora da integração neuromotora do idoso em executar com destreza tarefas que exijam as habilidades manuais e as capacidades coordenativas, onde os mesmos obtiveram um feedback positivo da melhora dos seus índices de percepção de execução com êxito das tarefas impostas no transcorrer desse processo, levando-os a se beneficiar nas suas atividades da vida diária, com ascensão da independência funcional.

Assim recomenda-se que sejam realizadas diversas pesquisas avaliando os resultados e suas intervenções através de programas ludomotores em diferentes variáveis das valências físicas que possam intervir no declínio da capacidade motora e funcional do idoso.

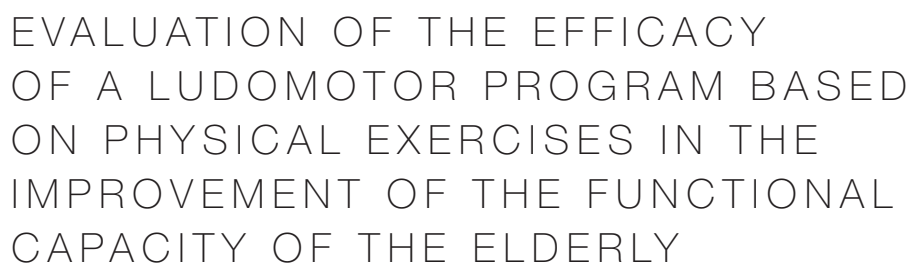

abstract

This study had the aim to evaluate the effectiveness of a ludomotor program around the difficult of the elderly to execute skillfully, in terms of speed and coordination, some activities of daily life that require their functional capacity. This study was compound by a hundred elderly of both sex, with age between 72 and 75 years old, registered at the "Happy Elderly" program in Rio de Janeiro. For the success of this research, all participants were submitted to Protocol of Manual Abilities (PMA). After that, the participants were submitted to a Coordination Test $(C T)$. You can obtain significant results through comparisons of the Protocol Manual Abilities that show an average performance equal to 11,71 seconds in the pre test and a performance of 10,60 seconds in the post test, as the coordination test achieved an average score of 11,29 seconds at the pre test and a score of 9,84 seconds in the post test, showing the efficiency of the intervention through the physical exercises program offered to these individuals. Thus, we can affirm the effectiveness of a ludomotor program of 
physical exercises in the improving of neuromotor integration of the elderly to execute skillfully tasks that require manual ability and coordinative capabilities: they achieved a positive feedback of the improvement of their sucessful executional perception indices in tasks imposed in the course of this process, causing them a benefit in their daily activities with the rise of functional independence.

keywords

Ludomotor Program. Functional Capacity. Elderly.

\author{
referências
}

ANDREOTTI, Rosana Aparecida; OKUMA, Silene Surime. Validação de uma bateria de testes de atividades da vida diária para idosos fisicamente independentes. Revista Paulista de Educação Física, São Paulo, v. 13, n. 1, p. 46-66, jan./jun. 1999.

BRUIN, Ender D. et al. Quantification of everyday motor function in a geriatric population. Journal of Rehabilitation Research and Development, Washington, v. 44, n. 3, p. 417-428, May, 2007

COHEN-MANSFIELD, Jiska; MARX, Maury S.; GURALNIK, Jackson M. Comparison of exercise models in an elderly population. Aging Clinical and Experimental Research, Milano, v. 18, n. 4, p. 312-319, Aug. 2006.

COHEN-MANSFIELD, Jiska; SHMOTKIN, Dov; GOLDBERG, Shira. Predictors of Longitudinal Changes in Older Adults' Physical Activity Engagement. Joumal of Aging and Physical Actrivity, Champaign, v. 18, n. 2, p. 141-157, Apr. 2010.

DIAS, Viviane Kawano; DUARTE, Priscila Sguassabia Ferreira. Idoso: níveis de coordenação motora sob prática de atividade física generalizada. Revista Digital, Buenos Aires, v. 10, n. 89, out. 2005. Disponível em: <http://mww.efdeportes.com/>. Acesso em: 24 maio 2013.

DOMINGUES, Flávia Maria; SANTOS, Flávia Costa Pinto. Manejo da bola da ginástica rítmica como estímulo ao desenvolvimento da destreza de mãos e dedos e velocidade de mãos e braços em adultos e idosos. Movimentum, Ipatinga, v. 1, p. 1-11, ago./ dez. 2006.

GALLAGHER, Nancy Ambrose et al. Neighborhood Factors Relevant for Walking in Older, Urban, African American Adults. Joumal of Aging and Physical Activity, Champaign, v. 18, n. 1, p. 99-115, Jan. 2010.

LEE, Young-Shin; LAFFREY, Shirley Cloutier. Predictors of physical activity in older adults with borderline hypertension. Nursing Research, Minneapolis, v. 55, n. 2, p. 110-120, Apr. 2006.

LIMA, Alisson Padilha de; DELGADO, Evaldo Inácio. A melhor idade do Brasil: aspectos biopsicossociais decorrentes do processo de envelhecimento. Ulbra e Movimento (REFUM), Ji-Paraná, v. 1, n. 2, p. 76-91, set./out. 2010.

LIMA, Alisson Padilha de et al. Uma avaliação da eficácia de um programa neuromotor de exercícios físicos para idosos. Brazilian Journal of Biomotricity, Nova Iguaçu, v. 5, n. 1, p. 26-33, mar. 2011. 
MATHEWS, Ana E. et al. Older Adults' Perceived Physical Activity Enablers and Barriers: A Multicultural Perspective. Journal of Aging and Physical Activity, Champaign, v. 18, p. 119-140, Apr. 2010

MONTE, Aurinice Sampaio Irene et al. Ginástica recreativa: um paradigma para a coordenação motora do idoso. Fitness e performance Joumal, Rio de Janeiro, v. 5, n. 4, p. 232-235, jun. 2006.

NICOLAl, Simone et al. Day-to-Day Variability of Physical Activity of Older Adults Living in the Community. Journal of Aging and Physical Activity, Champaign, v. 18, n. 1, p. 75-86, Jan. 2010.

OSNESS, Way H. et al. Functional Fitness Assessment for Adults Over 60 Years (A Field Based Assessment). Reston: American Alliance for Health, Physical Education, Recreation and Dance, 1990.

PHILLIPS, Edward M. et al. Interruption of Physical Activity Because of Illness in the Lifestyle Interventions and Independence for Elders Pilot Trial. Journal of Aging and Physical Activity, Champaign, v. 18, n. 1, p. 61-74, Jan. 2010.

SIVAN, Manoj; SAWYER, Ceinwen; BROWN, James. The role of exercise therapy in the secondary prevention of falls in elderly people. International Musculoskeletal Medicine, Leeds, v. 32, n. 4, p. 168-172, Dec. 2010.

Recebido: 04/11/2011

1a Revisão: 07/02/2013

$2^{a}$ Revisão: 11/04/2013

Aceite Final: 22/04/2013

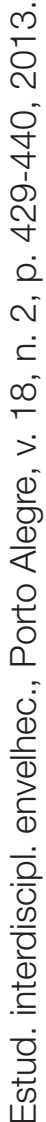

